\title{
Evaluating Digital Angles by a Parallel Diffusion Process
}

\author{
V. Cantoni ${ }^{+}$, L. Cinque ${ }^{\circ}$, S. Levialdi ${ }^{\circ}$, L. Lombardi ${ }^{+}$ \\ 'Dipartimento di Scienze dell'Informazione, Universita" "La Sapienza" di Roma \\ Via Salaria 113, 00198 Roma, Italy \\ +Dipartimento di Informatica e Sistemistica, Universita' di Pavia \\ Via Abbiategrasso 209, 27100 Pavia, Italy
}

\begin{abstract}
This paper describes a new method that uses the diffusionlike process to classify angles in a image. The proposed method exploits the curvature value of a contour line to characterize the correspondent angle. The effectiveness of the new method is illustrated with the results of experiments for a number different angle types.
\end{abstract}

\section{Introduction}

The purpose of low-level vision is to extract useful information from the images. To date, differential edge detectors have proven the best tools for this purpose. Although primitive features such as edge and lines are vital visual clues, intersection of edges such as corners (angles) and junctions, which are commonly refereed to as $2 \mathrm{D}$ features, provide richer informations for examining frame-toframe displacement characteristics of images. In general, edge detectors do not make good corner detectors because they give reduced output at corners, and because of this, considerable research has been specifically directed towards isolating corners points.

Our interest in the problem of corner detection arises from the desire to use the curvature value of a contour line to classify the correspondent angle. Many methods have been proposed in the literature for corner detection. Some of these are applied on digital curves or region boundaries after a prior segmentation of the image [1, ?]. More recent approaches are focused on developing methods which could detect corners by operating directly on the gray level images $[3,4]$. A detailed review and a comparative study of gray level corner detectors can be found in $[5,6]$.

In this paper we describe a new method the uses the diffusion-like process to classify angles. The proposed method exploits the curvature value of a contour line to characterize the correspondent angle. The diffusion approach, our implementation and how we use this method to classify angles is described in the next sextion. Results of experiments for selecting a safe set of thresholds for angles classification are carried out in section 3 , and we draw conclusions in section 4 . 


\section{Description of Basic Method}

Our method for the characterization of the shape [7], is based on the analogy with a diffusion process computing numerical values which reflect the object contour curvature.

In our approach the diffusion process acts on a physical object having the same shape as the digital object on which the contour must be analyzed. The contour of the object is heated, instantaneously at time $t=0$, at a given energy value. After a time $t=t_{f}$ the temperature of the contour is measured for each pixel; if we assume the object is,thermally insulated from the background, the value of contour pixels can be used to generate a shape-related code.

In our implementation the procedure starts by assigning to all the contour elements of the digital object to be labeled, an arbitrary (non-zero) value which initializes the diffusion process, while all the other elements are set to zero.

At the end of the process, the contour elements that preserve high values correspond to local convexities and those in which a sharp decrement is produced correspond to local concavities.

To detect these features it is necessary to stop the diffusion process at a certain stage. When the simulated diffusion process is stopped, the values are distributed along the contour depending on the local shape configuration; these values will be used for contour labeling.

\subsection{Angle characterization}

We have applied the proposed method to classify angles. For a given number of iterations a set of thresholds may be chosen so as to associate values exceeding such thresholds with angle labels. As an example the method has been applied on a square with a hole (figure 1), the result is presented in figure 2. A uniform gray scale was chosen to show the different values obtained on the border: white represents a high value and black a low one.

\section{Experimental results}

The purpose of this section is to show that we can use the proposed method for classifying angles. We have performed experiments on images with known characteristics.

The experimental protocol involved the application of the procedure to each image with different values, for diffusion coefficient $D$ and the number of steps $N$, as shown in Table 1 . Test images are circular sectors with different angles $\theta$ $(\theta=30,60,90, \ldots, 330)$ at different spatial orientations.

The results of our experiments are shown in a set of tables (one for each value of $D$ and $N$ ) in which for each angle the minimum, the medium and the maximum values are given (see Table 2,3). These values describe the range associate to the angle. 


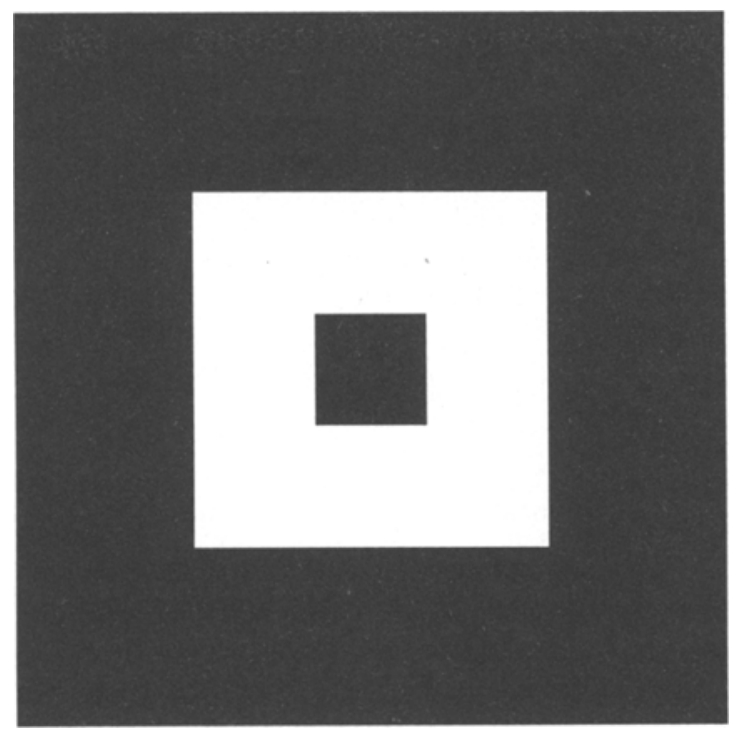

Fig. 1. Test sample object.

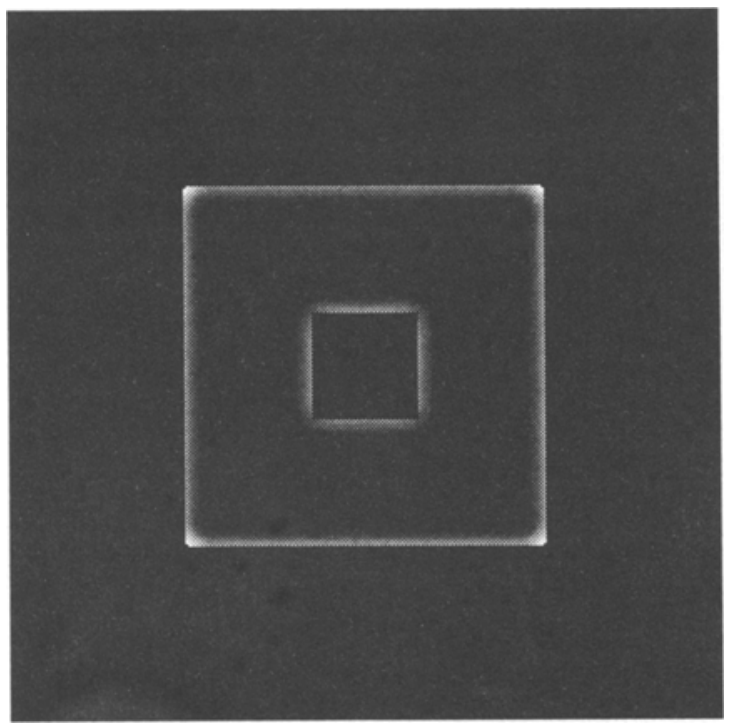

Fig. 2. Result of the diffusion procedure on the object of figure 1 . White represents a high value and black a low one. 


\begin{tabular}{|l|rrrrr|}
\hline$D$ & \multicolumn{5}{|c|}{$N$} \\
\hline 0.05 & 20 & 30 & 40 & 50 & 60 \\
0.025 & 40 & 60 & 80 & 100 & 120 \\
0.0125 & 80 & 120 & 160 & 200 & 240 \\
\hline
\end{tabular}

Table 1. Set of parameter values used in experiments. For each diffusion coefficient $D$ five different number of steps $N$ have been used. Note that prodocts $N \times D$ are the same on each row.

From the set of experimentally tables we give only two tables as an example, these represent two meaningful cases. The first table represents a situation where we cannot recognize safely classes of angles because some ranges of values overlap. This is the case of the ranges for angles $60^{\circ}$ and $90^{\circ}$ respectively $[1927,2201]$ and $[1980,2285]$; these values are obtained with a diffusion coefficient $D=0.025$ and with a number of iterations $N=40$. The second table shows instead a positive result where we can select safe thresholds; in fact all the range values are disjoint. These values have been computed with $D=0.025$ and $N=120$.

\begin{tabular}{|r|ccc|}
\hline$\theta$ & Min & Mean & Max \\
\hline 30 & 1730 & 1764 & 1815 \\
60 & 1927 & 2013 & 2201 \\
90 & 1980 & 2166 & 2285 \\
120 & 2305 & 2411 & 2548 \\
150 & 2641 & 2743 & 2833 \\
180 & 2936 & 3301 & 3596 \\
210 & 3766 & 3966 & 4362 \\
240 & 4082 & 4369 & 4379 \\
270 & 4969 & 5083 & 5246 \\
300 & 5607 & 5931 & 6363 \\
330 & 6524 & 6992 & 7950 \\
\hline
\end{tabular}

Table 2. Experimental results with $D=0.025$ and $N=40$.

A global view of all experiments are plotted in figures 3,4 .

The graphs, as expected, are quite similar the only difference is in the width of errorbars; infact growing the number of steps the range of values associate with each angle becomes smaller.

Our final scope is to determine a set of thresholds that allow us to safely recognize eleven different angles $(30,60, \ldots, 330)$, having disjoint ranges. As 


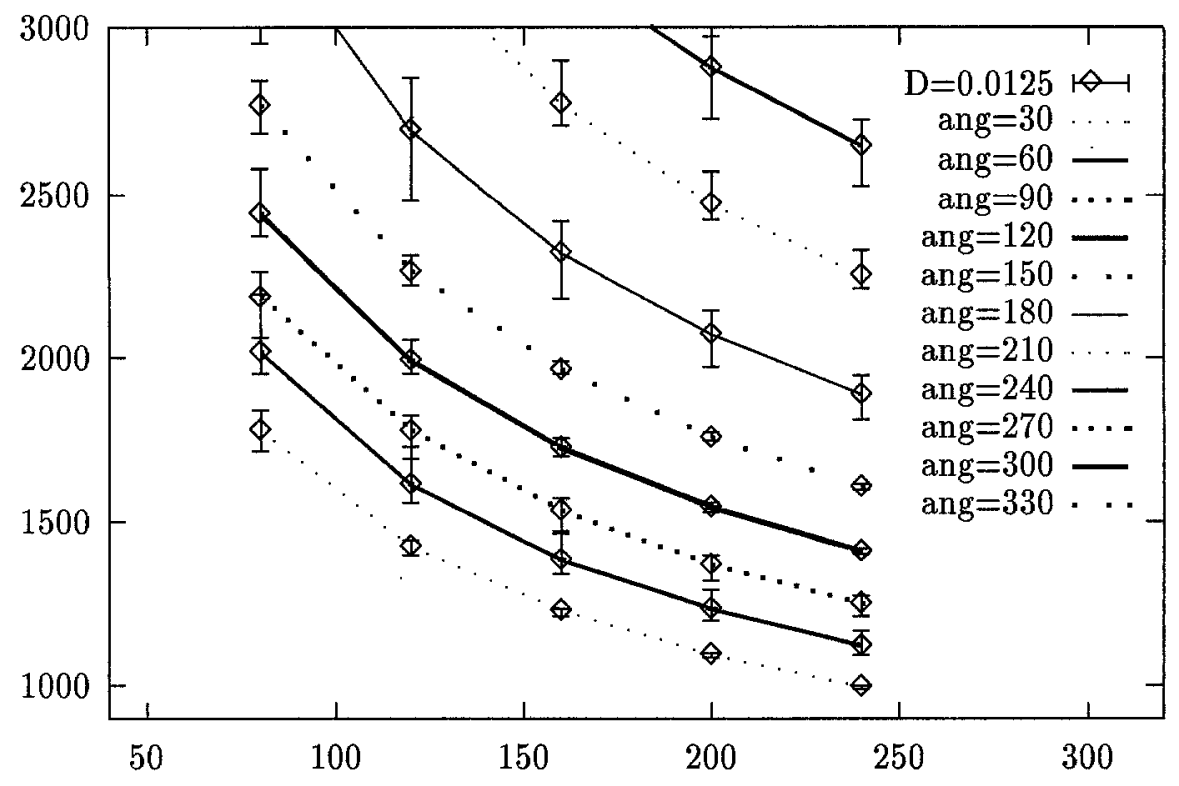

Fig. 3. Results of our procedure for different numbers of steps and $D=0.0125$.

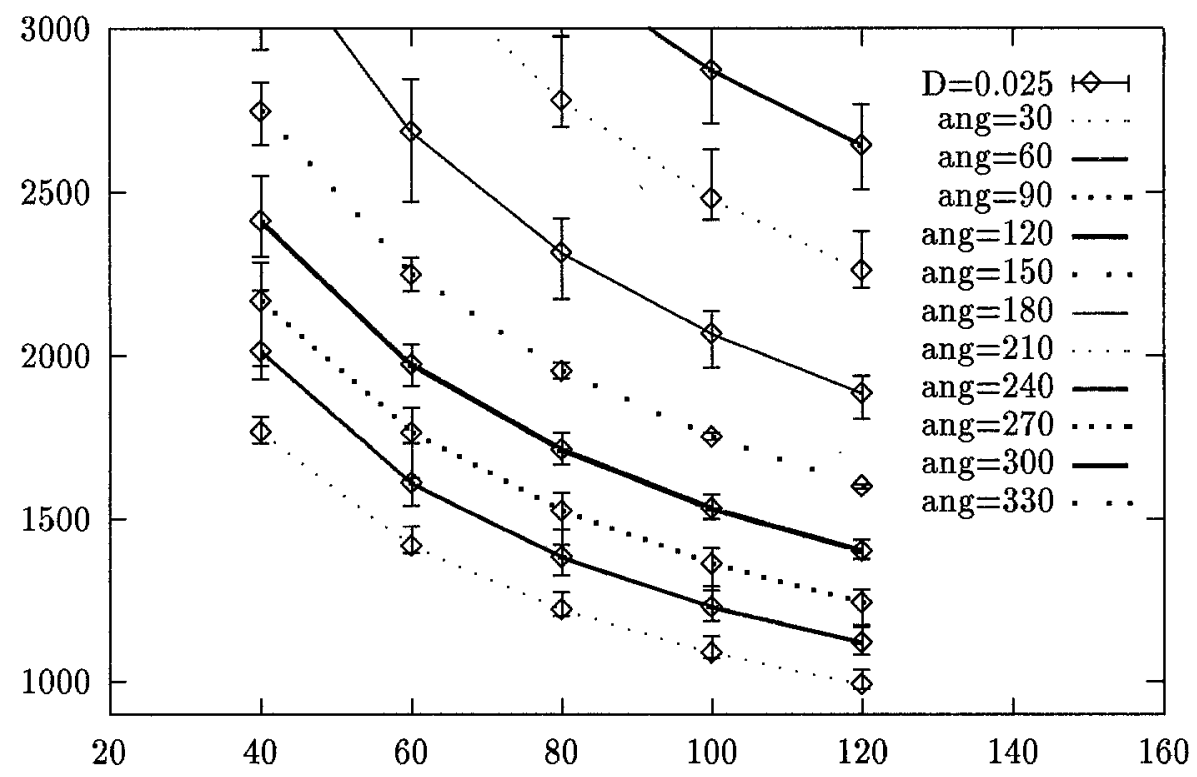

Fig. 4. Results of our procedure for different numbers of steps and $D=0.025$. 


\begin{tabular}{|r|rrr|}
\hline$\theta$ & Min & Mean & Max \\
\hline 30 & 978 & 992 & 1037 \\
60 & 1083 & 1118 & 1168 \\
90 & 1173 & 1240 & 1281 \\
120 & 1375 & 1398 & 1436 \\
150 & 1593 & 1597 & 1607 \\
180 & 1805 & 1885 & 1939 \\
210 & 2208 & 2259 & 2380 \\
240 & 2506 & 2639 & 2767 \\
270 & 3188 & 3241 & 3301 \\
300 & 4074 & 4180 & 4315 \\
330 & 5203 & 5638 & 6655 \\
\hline
\end{tabular}

Table 3. Experimental results with $D=0.025$ and $N=120$.

shown in the graphs a set of possible solutions are $D=0.025$ and $N \geq 120$. Safe results can be obtained using a high number of steps, the drawback is a higher computational cost. A good compromise is represented by the couple $D=0.0125, N=240$.

\section{Conclusions}

In this paper we have presented a diffusion based technique for angle measurement. We have shown that extrema of the profile of the heat distribution along the contour gives good clues about the position and width of the correspondent angle. The proposed method is suitable to highly parallel implementations, is invariant for rotation and partially insensitive to noise.

\section{References}

1. W. S. Rutkowski and A. Rosenfeld, "A comparison of corner detection techniques for chain-coded curves," TR-623, CS Center, Uni. of Maryland (1978).

2. X. Xie, R. Sudhakar and H. Zhuang, "Corner detection by a cost minimization approach," Pattern Recognition 26, 1235-1243; (1993).

3. L. Kitchen, and A. Rosenfeld, "Gray level corner detection," Pattern Recognition Lett. 1, 95-102, (1982).

4. R. M. Haralick and L. G. Shapiro, Computer and Robot Vision, Vol. I, AddisonWesley. Reading, Massachusetts (1992).

5. J. A. Noble, "The geometric structure of images," MSc Report. Oxford UK (1987).

6. F. Eryurtlu and J. Kittler, "A comparative study of gray level corner detectors," Signal processing VI:Theories and Appications. Elsevier Sci. Publ., (1992).

7. L. Cinque and L. Lombardi, "Shape description and recognition by a multiresolution approach," Image and Vision Computing. (to appear). 